\title{
Primordial Nucleosynthesis with a background magnetic field
}

\author{
Yudong Luo ${ }^{1,2, *}$, Toshitaka Kajino ${ }^{1,2,3}$, Motohiko Kusakabe ${ }^{3}$, and Michael A Famiano ${ }^{4}$ \\ ${ }^{1}$ National Astronomical Observatory of Japan,2-21-1 Osawa, Mitaka, Tokyo 181-8588, Japan \\ ${ }^{2}$ Department of Astronomy, Graduate School of Science, The University of Tokyo, 7-3-1 Hongo, \\ Bunkyo-ku, Tokyo 113-0033, Japan \\ ${ }^{3}$ School of Physics and Nuclear Energy Engineering and International Research Center for Big-Bang \\ Cosmology and Element Genesis, Beihang University, 37, Xueyuan Rd., Haidian-qu, Beijing 100083 \\ China \\ ${ }^{4}$ Department of Physics, Western Michigan University, Kalamazoo, Michigan 49008,USA
}

\begin{abstract}
We present our recent detailed calculation of the impacts from a background magnetic field on Big Bang Nucleosynthesis (BBN). Namely, the magnetic field impacts on the electron-positron thermodynamics, time temperature relation and the screening potential of the early Universe. Most interestingly, we investigated the electron-positron relativistic screening potential with the background magnetic field, such potential might lead to a non trivial effect on the electron capture reaction which could finally affect the neutron to proton ratio.
\end{abstract}

\section{Introduction}

In the standard big bang nucleosynthesis (BBN) model, the only free parameter is the baryonto-photon ratio $(\eta)$, which is considered as a homogeneous quantity. Currently $\eta$ is well determined from the power spectrum of the cosmic microwave background temperature fluctuations [1]. The previous study [2] introduced a constant scale invariant primordial magnetic field (PMF) strength within a co-moving radius $1 \mathrm{Mpc}$ during the BBN epoch. However, smaller scale of the PMF fluctuations inside the co-moving horizon is expected to survive during the BBN epoch [3]. It is possible to have sub-horizon PMF strength during neutrino decoupling epoch which can consequently lead to an energy density inhomogeneity in the early Universe. Previous study have already encoded the statistical distribution of such energy density into the BBN network calculation [4]. However, such treatment cannot represent the systematic dynamical PMF evolution including fluctuation calculation. In order to do that, a multi-zone BBN with dynamical evolution of PMF calculation is required. In this study, we firstly try to have a comprehensive understanding of the magnetic field impacts on the particles interaction as well as on the hot plasma of the early Universe.

\section{Magnetic field impacts on BBN}

The primordial magnetic field can affect the BBN from both microscopic and macroscopic perspective. The most sensitive particles of magnetic field are electron and positron since

\footnotetext{
*e-mail: ydong.luo@nao.ac.jp
} 
they have larger magnetic momentum compared with charged baryons. The thermodynamics of $e^{ \pm}$will be affected via Landau quantization [5]:

$$
\begin{aligned}
n_{e^{ \pm}} & =\frac{e B}{(2 \pi)^{2}} \sum_{n=0}^{\infty}\left(2-\delta_{n 0}\right) \int_{-\infty}^{\infty} f_{F D}\left(E_{B}, T_{\gamma}\right) d p_{z} \\
\rho_{e^{ \pm}} & =\frac{e B}{(2 \pi)^{2}} \sum_{n=0}^{\infty}\left(2-\delta_{n 0}\right) \int_{-\infty}^{\infty} E_{B} f_{F D}\left(E_{B}, T_{\gamma}\right) d p_{z} \\
P_{e^{ \pm}} & =\frac{e B}{(2 \pi)^{2}} \sum_{n=0}^{\infty}\left(2-\delta_{n 0}\right) \int_{-\infty}^{\infty} \frac{E_{B}^{2}-m_{e}^{2}}{3 E_{B}} f_{F D}\left(E_{B}, T_{\gamma}\right) d p_{z}
\end{aligned}
$$

where $f_{F D}$ is the Fermi-Dirac distribution, $T_{\gamma}$ is the photon temperature, $E_{B}=p_{z}^{2}+m_{e}^{2}+2 n e B$ (here after, we set $\hbar=c=k_{B}=1$ ). $n_{e^{ \pm}}, \rho_{e^{ \pm}}$and $P_{e^{ \pm}}$represents the number density, the energy density and the pressure of $e^{ \pm}$in the early Universe.

Another microscopic affect from the PMF is that it can change the screening potential of the electron-positron plasma. The screening potential can be treated as a correction term of the Coulomb potential for the low density plasma [6]:

$$
V_{C}^{s c r}=\frac{Z_{1} Z_{2} e^{2}}{r} \exp \left(-\frac{r}{\lambda_{D}}\right) \simeq V_{C}^{\text {bare }}-\frac{Z_{1} Z_{2} e^{2}}{\lambda_{D}},
$$

where $\lambda_{D}$ is the Debye length. The last approximation is valid for the low density plasma where the $\lambda_{D} \gg r$ (i.e, weak screening), in other words, the weak screening effect can shift the bare Coulomb potential energy by amount of $\Delta_{V}=Z_{1} Z_{2} e^{2} / \lambda_{D}$. The contribution to the Debye length from the relativistic $e^{ \pm}$plasma is given by Schwinger-Dyson equation of photon propagator [7]. However, with the magnetic field background, shown by Eq. 13 , the electron's thermodynamical properties are modified, therefore after summing over the electron spin and all the Landau levels, the Debye length is given by

$$
\frac{1}{\lambda_{D}^{2}}=4 \pi e^{2} \frac{\gamma m_{e}^{3}}{2 \pi^{2}} \frac{\partial}{\partial \mu} \sum_{n=0}^{\infty}\left(2-\delta_{n_{0}}\right) \int_{0}^{\infty} d p_{z}\left[\frac{1}{1+\exp \left(E_{n}-\mu\right) / k T}-\frac{1}{1+\exp \left(E_{n}+\mu\right) / k T}\right],
$$

where $\gamma=B / B_{c}$ and $\mu$ is the electron chemical potential. Before weak interaction decoupling, neutrons and protons are indistinguishable to each other due to the Nuclear Statistical Equilibrium (NSE) of the weak interaction $n \leftrightarrow p$, but in previous studies [8,9] it was pointed out that the effects PMF contribution to the weak interaction is negligible. However, since the screening potential shows non-trivial correlation with PMF strength, the weak interaction rate is expected to be deviated from the circumstance without PMF. This is true particularly for the electron capture reaction $\left(p+e^{-} \rightarrow n+v_{e}\right)$ because of the Coulomb repulsion between a proton and an electron, is expected to be deviated from the circumstance without PMF. Follow Eq. 4, the weak screening changes the potential energy. For electron capture reaction, such effect can be treated as the deviation of the electron kinetic energy from $E$ to $E^{\prime}=E-\Delta_{V}$ therefore, the reaction rate is

$$
\begin{array}{r}
\lambda_{p e^{-} \rightarrow n v_{e}}^{B s c r}=\frac{G_{F}^{2} T_{\gamma}^{2}\left(g_{V}^{2}+3 g_{A}^{2}\right)}{2 \pi^{3}} z \sum_{n=0}^{\infty}\left(2-\delta_{n 0}\right) \times \int_{\sqrt{1+4 \gamma n}}^{\infty} \frac{\epsilon^{\prime} d \epsilon^{\prime}}{\sqrt{\epsilon^{\prime 2}-(1+4 \gamma n)}} \\
\frac{\left(\epsilon^{\prime}+q\right)^{2}}{1+\exp \left(\epsilon^{\prime} z_{e}-\phi_{e}\right)} \frac{\exp \left(\epsilon^{\prime} z_{e}-\phi_{e}\right)}{1+\exp \left[\left(\epsilon^{\prime}+q\right) z_{v}-\phi_{\nu}\right]} .
\end{array}
$$

Here, $q=\left(m_{n}-m_{p}\right) / m_{e} c^{2}, z=m_{e} c^{2} / k T$ and $\epsilon^{\prime}=\epsilon-\Delta_{V} / m_{e} c^{2}$ where $\epsilon=E / m_{e} c^{2}$. We show the ratio of the reaction rate between the case with screening effect and without in Fig. 1 . We 


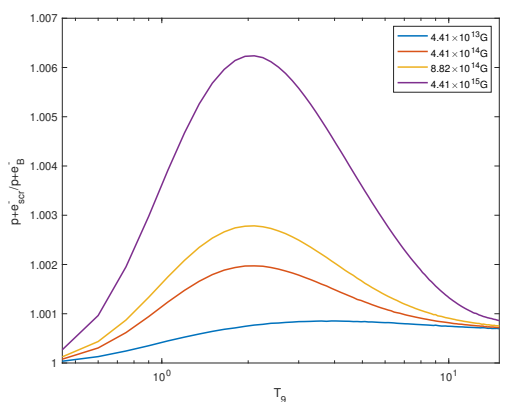

Figure 1. The screening correction to the $p e^{-} \rightarrow n v$ reaction rate as function of $T_{9}$ under different PMF strength. Y-axis is the ratio between the reaction rate with screening correction and without the correction.

also compare different PMF strength here. As the PMF strength becomes stronger, its impact on the Debye length is amplified, which lead to a enhancement of $p+e^{-} \rightarrow n+v_{e}$ finally.

After neutrino decoupling, neutrinos became free particles so that their temperature decrease as $T_{v} \propto R^{-1}$, where $R$ is the scale factor of the Universe. On the other hand, the energy conservation requires $d\left[\left(\rho_{e m}+P_{e m}\right) R^{3}\right]=R^{3} d P_{e m}$, with $\rho_{e m}=\rho_{e}+\rho_{\gamma}$ and $P_{e m}=P_{e}+P_{\gamma}$ (here $\rho_{e}$ and $P_{e}$ are the summation quantity for both electron and positron), after taking into account the magnetic field, the Hubble expansion rate now can be written as

$$
H=\frac{\dot{R}}{R}=\sqrt{\frac{8 \pi G \rho_{t o t}}{3}},
$$

where $\rho_{t o t}$ includes $\rho_{P M F}$ as well. The photon temperature as a function of time with the existence of magnetic field becomes [9]

$$
\frac{d T_{\gamma}}{d t}=-3 H \frac{\rho_{e m}+P_{e m}}{d \rho_{e m} / d T_{\gamma}},
$$

where the electron energy density and pressure are described using the formalism in Eq.1-3 as a function of magnetic field strength.

\section{Acknowledgments}

This work is supported by JSPS KAKENHI Grant No.19J22167.

\section{References}

[1] Planck Collaboration, A\&A 594, 13 (2016)

[2] D.G.Yamazaki and M.Kusakabe, Phys. Rev. D 86(12), 123006 (2012)

[3] A.D.Dolgov and D.Grasso, Phys. Rev. Lett. 88(1), 279 (2001)

[4] Y.Luo, T.Kajino, M.Kusakabe and G.J.Mathews, ApJ 872, 172 (2019)

[5] M.Kawasaki and M.Kusakabe, Phys. Rev. D 86(6), 063003 (2012)

[6] B.Wang, C.A.Bertulani and A.B.Balantekin, Phys. Rev. C 83, 018801 (2011)

[7] M.A.Famiano, A.B.Balantekin and T.Kajino Phys. Rev. C 93, 045804 (2016)

[8] B.Cheng, D.Schramm and J.Truran, Phys. Lett. B 316, 521 (1993)

[9] P.Kernan, G.Starkman and T.Vachaspati, Phys. Rev. D 54, 7207 (1996) 